\title{
Efficient Bypass of Mutations in Dysferlin Deficient Patient Cells by Antisense-Induced Exon Skipping
}

\author{
Nicolas Wein, ${ }^{1 \dagger}$ Aurélie Avril, ${ }^{2 \dagger}$ Marc Bartoli, ${ }^{1}$ Cyriaque Beley, ${ }^{2}$ Soraya Chaouch, ${ }^{2}$ Pascal Laforêt, ${ }^{3}$ Anthony Behin, ${ }^{3}$ \\ Gillian Butler-Browne, ${ }^{2}$ Vincent Mouly, ${ }^{2}$ Martin Krahn, ${ }^{1,4}$ Luis Garcia, ${ }^{2 \ddagger}$ and Nicolas Lévy ${ }^{1,4 * \ddagger}$ \\ 'Université de la Méditerranée, Inserm UMR_S 910 "Génétique Médicale et Génomique Fonctionnelle," Faculté de Médecine de Marseille, \\ France, ${ }^{2}$ Université Pierre et Marie Curie/Paris 6/Inserm UMR_S 974, CNRS UMR 7215, Institut de Myologie, Groupe Hospitalier Pitié-Salpêtrière, \\ Paris, France; ${ }^{3}$ Centre de référence de pathologie neuromusculaire Paris Est, Groupe Hospitalier Pitié-Salpêtrière, AP-HP, Paris, France; \\ ${ }^{4}$ Département de Génétique Médicale, Hôpital d'enfants de la Timone, AP-HM, Marseille, France
}

ABSTRACT: Mutations in DYSF encoding dysferlin cause primary dysferlinopathies, autosomal recessive diseases that mainly present clinically as Limb Girdle Muscular Dystrophy type 2B and Miyoshi myopathy. More than 350 different sequence variants have been reported in DYSF. Like dystrophin, the size of the dysferlin mRNA is above the limited packaging size of AAV vectors. Alternative strategies to AAV gene transfer in muscle cells must then be addressed for patients. A gene therapy approach for Duchenne muscular dystrophy was recently developed, based on exon-skipping strategy. Numerous sequences are recognized by splicing protein complexes and, when specifically blocked by antisense oligoucleotides $(\mathrm{AON})$, the corresponding exon is skipped. We hypothesized that this approach could be useful for patients affected with dysferlinopathies. To confirm this assumption, exon 32 was selected as a prioritary target for exon skipping strategy. This option was initially driven by the report from Sinnreich and colleagues of a patient with a very mild and late-onset phenotype associated to a natural skipping of exon 32. Three different antisense oligonucleotides were tested in myoblasts generated from control and patient MyoD transduced fibroblasts, either as oligonucleotides or after incorporation into lentiviral vectors. These approaches led to a high efficiency of exon 32 skipping. Therefore, these results seem promising, and could be applied to several other exons in the DYSF gene. Patients carrying mutations in exons whose the in-frame suppression has been proven to have no major consequences on the protein function, might benefit of exonskipping based gene correction.

Hum Mutat 30:1-7, 2009. (c) 2009 Wiley-Liss, Inc.

KEY WORDS: DYSF; dysferlin; exon-skipping; gene therapy; myoblast

\section{Introduction}

Dysferlinopathies are autosomal recessive diseases covering a wide spectrum of phenotypes, mainly Limb Girdle Muscular Dystrophy type 2B (LGMD2B; MIM\# 253601) [Bashir et al., 1998] and Miyoshi myopathy (MM; MIM\# 254130) [Liu et al., 1998], but ranging from isolated HyperCPKemia to severe disability [Nguyen et al., 2007; Urtizberea et al., 2008]. Onset is usually in the second decade of life, with a progressive course of muscle weakness and possible wheelchair dependency after decades of disease progression, or following a more severe and rapid evolutionary course in some patients.

Dysferlinopathies are caused by mutations in the large-sized gene encoding dysferlin (DYSF; MIM\# 603009, 2p13, GenBank NM_003494.2) [Bashir et al., 1998; Liu et al., 1998], a 237-kDa plasma membrane protein that plays a central role in sarcolemmal repair [Bansal et al., 2003]. DYSF has a large mutational spectrum and more than 350 different sequence variants have been reported (Leiden Muscular Dystrophy pages(C), www.dmd.nl), mainly point mutations and small insertions/deletions [Krahn et al., 2009a], but also diseasecausing exonic deletions or duplications [Krahn et al., 2009b].

As for most muscular dystrophies, there is currently no curative treatment available for dysferlinopathies. Recently, transcript rescue strategies have been developed as therapeutic approaches in dystrophinopathies [Goyenvalle et al., 2004], and clinical trials are underway [Heemskerk et al., 2009]. Transcript rescue is based on the use of antisense-molecules to induce the targeted deletion, at the pre-mRNA level, of one or several exons carrying diseasecausing mutations, while maintaining an open reading-frame [Aartsma-Rus et al., 2004; Goyenvalle et al., 2004; Lu et al., 2005]. However, such "exon-skipping" strategies are applicable only for proteins for which part of the amino acid (aa) sequence can be deleted without important deleterious impact on the overall function. Noteworthy, dysferlin contains six or seven predicted C2 domains (www.expasy.ch/), implicated in calcium and phospholipid binding, raising the question of possible functional compensation for at least part of this protein. However, although exon-skipping is technically feasible regardless of the gene's structure, its pertinence must be carefully addressed. A comprehensive exon map including the phasing of dysferlin is provided in Figure 1A. In DYSF, several in frame deletions of exons resulting from splice site mutations (exon 25 [Therrien et al., 2006] and 49 [De Luna et al., 2007]) have been reported as being associated to phenotypes ranging from typical to severe, clearly indicating that these exons are not proper targets for an exon skipping strategy. 

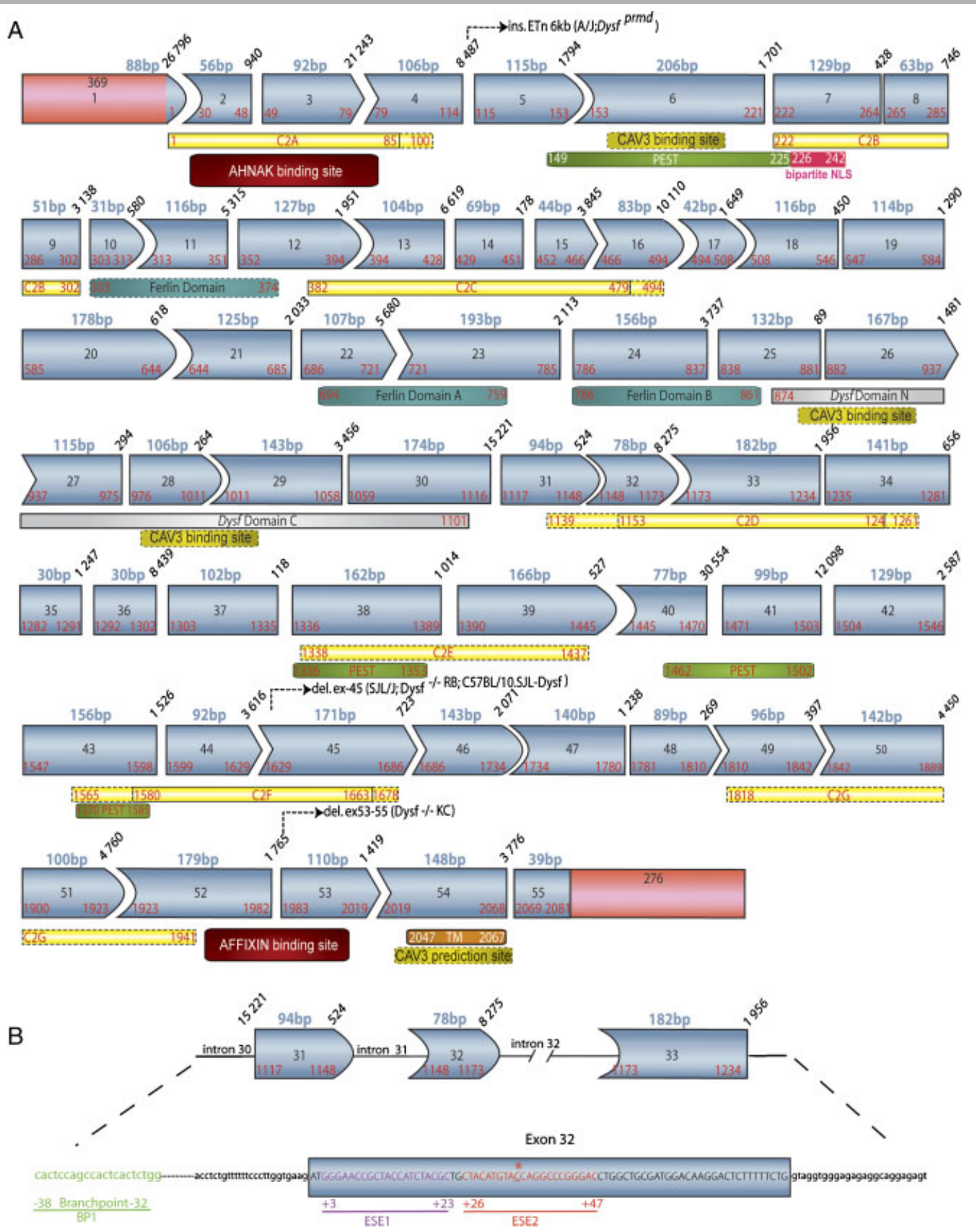

Figure 1. Human dysferlin exon map and phasing. A: Representation of the 55 exons of the human dysferlin gene (blue). Red boxes correspond to the $5^{\prime}$ and $3^{\prime}$ UTR. On top of each exon, the length in base pairs is indicated. Inside the boxes the position of the first and last residue is written in red. Below each exon the bioinformatics predictions of protein domains are represented (ScanProsite solid line and Pfam dotted line). The intron lengths are mentioned between the exons. The genetic defect in the dysferlin gene of three mouse models are depicted on the scheme. The phasing was performed using the UMD-DYSF database, a specific database generated on a similar model, compared to UMD-DMD [Tuffery-Giraud et al., 2009]. B: Schematic representation of exon 32 and surrounding sequences. The selected 2'0-methyl phosphorothioate oligonucleotide (AON) are indicated below the sequence. These AON blocked either the branching point (BP) or exonic splicing enhancers (predicted using Human Splicing Finder) [Desmet et al., 2009] (ESE1) or using ESE finder 3.0 (ESE2). The asterisk indicates the mutated nucleotide in patient F1-38-1-2.

Moreover, apart from the C2 domains whose functional redundancy has been at least partially demonstrated [Krahn et al., 2008], several domains are unique, and their suppression would be undoubtedly deleterious. In particular, the unique terminal transmembrane (TM) domain is encoded by a specific sequence in exon 54 and, although a possible frame rescue could be expected after skipping both exons 53 and 54, these exons should be definitively excluded as possible skipping targets. In contrast, and most interestingly, Sinnreich and colleagues [2006] reported a patient with a particularly mild phenotype of dysferlinopathy. This patient was the mother of two severely affected sisters, both homozygous for a dysferlin null mutation. Their mother was a compound heterozygous for the same 
null mutation, and in addition, carried a lariat branch point mutation in intron 31 leading to an in-frame skipping of exon 32. Because she presented with only a mild proximal weakness and remained ambulant even after 70 years of age, it is most likely that the exon 32 skipped allele may partially complement the null mutation in trans.

We thus considered the report from Sinnreich and colleagues [2006] as a "natural" proof of principle for an exon-skipping approach targeting exon 32 in dysferlinopathies. Assuming that the deletion of this exon has no major consequences on dysferlin function, its removal appears to be a valuable and pertinent therapeutic strategy in patients carrying nonsense or frameshifting mutations, the only types of mutations reported to date in DYSF exon 32 in 13 patients.

\section{Materials and Methods}

\section{Skin Fibroblast and Myoblast Cultures}

All experiments and biopsies were carried out in accordance with the ethical rules of the institutions involved. Patient fibroblast cultures were derived from a skin biopsy obtained from a patient affected with Miyoshi myopathy (Patient F1-38-1-2: compound heterozygous c.3477C $>$ A [exon 32, p.Tyr1159X] and c.5979dupA [exon 53, p.Glu1994ArgfsX3]). Mutational data are described using the nomenclature of the Human Genome Variation Society (www.hgvs.org/mutnomen). Nucleotide numbering reflects cDNA numbering with +1 corresponding to the A of the ATG translation initiation codon in the reference sequence (human DYSF, GenBank NM_003494.2), according to journal guidelines. The initiation codon is codon 1 .

Control myoblasts (CHQ) were derived from a quadriceps biopsy performed on a 5-day-old donor, as already described [Edom et al., 1994]. All culture media and reagents were obtained from Gibco (Invitrogen, Paisley, UK). Primary human cells were extracted from biopsies by enzymatic dissociation with collagenase 1A (SigmaAldrich, St. Louis, MO). Cells were expanded in the proliferation medium composed of DMEM (Gibco), 20\% fetal bovine serum (FBS; PAA Laboratories GmbH, Pasching, Austria), $100 \mu \mathrm{g} / \mathrm{mL}$ penicillin, $100 \mu \mathrm{g} / \mathrm{mL}$ streptomycin (Biowest, Nuaille, France), and L-glutamin (Gibco). At confluence, cells were cultured in the differentiation medium composed of DMEM supplemented with Glutamax and $1 \mathrm{mM}$ pyruvate sodium (Gibco), 2\% horse serum (Gibco), $10 \mu \mathrm{g} / \mathrm{mL}$ insulin (Sigma-Aldrich), $100 \mu \mathrm{g} / \mathrm{mL}$ apotransferrin (Sigma-Aldrich), $100 \mu \mathrm{g} / \mathrm{mL}$ penicillin, $100 \mu \mathrm{g} / \mathrm{mL}$ streptomycin, and L-glutamine [Chaouch et al., 2009].

\section{AONs and Transfection}

Exonic splicing enhancer (ESE) and exonic splicing silencer (ESS) sequences were identified by Human Splicing Finder [Desmet et al., 2009] and ESE finder 3.0 [Cartegni and Krainer, 2003; Smith et al., 2006]. The corresponding selected sequences are represented in Figure 1. All AONs were synthesized by Eurogentec (Seraing, Belgium) and contain 2'-O-methyl RNA and full-length phosphorothioate (PS) backbones. Fibroblast-derived myoblast cultures were transfected using Oligofectamin (Invitrogen) according to the manufacturer's instructions. Each AON was transfected twice at $10 \mu \mathrm{g} / \mu \mathrm{L}$ in at least two separate experiments. A control AON with a $5^{\prime}$ fluorescein label was used to ascertain optimal transfection, efficiencies were evaluated by counting the number of fluorescent nuclei (over $90 \%$ of all nuclei were labeled). RNA was isolated $48 \mathrm{hr}$ posttransfection.

\section{Vector Construction and Production}

U7 snRNA. The U7 DNA constructs were engineered from the previously described U7SmOPT; where the original Sm binding domain is replaced by an optimized U2 type binding domain [Gorman et al., 1998] and antisense sequence targeting the histone pre-mRNA is replaced by specific antisense sequences targeting splicing sequences within the human pre mRNA of dysferlin (Fig. 1). The constructions U7smOPT-ESE1, U7smOPT-ESE2, U7smOPT-ESE1-ESE2, and U7smOPT-ESE2-ESE1 fragments were subcloned into a plasmid pRRL-cPPT-mcs-WPRE, derived from the pRRL-cPPT-hPGK-eGFP-WPRE construct [Naldini, 1998]. Constructs were sequenced on an ABI3130xl Genetic Analyzer (Applied Biosystems, Carlsbad, CA) and analyzed with Sequencher software (Gene Codes Corporation, Ann Arbor, MI).

\section{MyoD}

The mouse MyoD cDNA was inserted into the tet-on inducible lentiviral construct previously described containing the tetresponsive transactivator rtTA2S-M2 inducing the MyoD expression in the presence of doxycycline [Barde et al., 2006].

\section{Production}

As previously described, lentiviral vectors pseudotyped with the VSV-G protein were produced by transient quadri-transfection into 293T cells (Coriell Cell Repository; Camden, NJ) and the viral titers (infectious particles) were determined by transduction of HCT116 cells and assayed by quantitative real-time PCR on genomic DNA [Charrier et al., 2005]. Titration of the four lentivirus is expressed as viral genome $/ \mathrm{mL}(\mathrm{vg} / \mathrm{mL}$ ) (ranging from $4.5 \times 10^{8}$ to $\left.2.4 \times 10^{9} \mathrm{vg} / \mathrm{mL}\right)$.

\section{Lentiviral Vector Transduction of Fibroblasts}

For Myo-D induction of fibroblasts, $2 \times 10^{3}$ to $6 \times 10^{3}$ cells $/ \mathrm{cm}^{2}$ were plated into gelatin B (Sigma) precoated wells. At about $70 \%$ confluence, 2 to $5.10^{9} \mathrm{vg} / \mathrm{mL}$ lentivirus were added into $500 \mu \mathrm{L}$ of DMEM medium, diluted $4 \mathrm{hr}$ later into $500 \mu \mathrm{L}$ of the proliferation medium. To induce MyoD expression, doxycycline $(10 \mu \mathrm{g} / \mathrm{mL})$ (Sigma) was added at about $80 \%$ confluence and subsequently, at full confluence, the proliferation medium was replaced by the differentiation medium supplemented with doxycycline $(10 \mu \mathrm{g} / \mathrm{mL})$. MyoD-induced expression of specific myogenic markers was evaluated by RT-PCR after doxycycline induction (data not shown). Transduction of fibroblast derived myoblasts and CHQ cells [Edom et al., 1994] with U7smOPT-ESE1, U7smOPT-ESE2, U7smOPTESE1-ESE2, and U7smOPT-ESE2-ESE1 lentiviral vectors was carried out using the same protocol [Chaouch et al., 2009].

\section{RNA Extraction and Classical and Nested PCR}

RNAs were extracted from cultured cells using RNeasy kit and mRNA were purified with a poly dT column (Qiagen, Courtaboeuf, France). One microgram of mRNA was reverse transcribed into cDNA with the Super-Script II RT (Invitrogen) primed by random hexamers according to the manufacturer. PCR amplifications were performed with $1 \mu \mathrm{g}$ of RT-product using primer pairs flanking the targeted exons (sequences available upon request) for 35 cycles. Dysferlin RNA exon skipping was analyzed by classical RT-PCR for AONs study and nested RT-PCR for U7 lentiviral transduction study. The products of PCR were separated by electrophoresis in a $1.5 \%$ agarose gel stained with ethidium 
bromide. The PCR product was then gel extracted using NucleoSpin extract II (Macherey-Nagel GmbH \& Co KG, Düren, Germany) and sequenced using the Big Dye Terminator Cycle Sequencing Kit (Applied Biosystem, Courtaboeuf, France).

\section{Results and Discussion}

\section{Identification of Target Sequences for Dysferlin Exon 32 Skipping}

We performed bioinformatics analyses to find targetable sequences within exon 32 of the DYSF gene and its surrounding intronic sequences. Various sequences such as exon splicing enhancers (ESE) are recognized by specific splicing proteins and are required to maintain exons within mRNAs. If some of these sequences are masked or absent, the targeted exon is skipped [Aartsma-Rus et al., 2004]. According to the bioinformatics analyses performed (www.umd.be/HSF/ and http://rulai.cshl.edu/ cgi-bin/tools/ESE3/esefinder.cgi?process = home) $\quad[$ Cartegni and Krainer, 2003; Desmet et al., 2009; Smith et al., 2006], two sequence regions containing clustered predicted ESE sites were identified, while a third sequence was identified as containing the lariat branch point in intron 31 . These sequences were selected to be further targeted with 2'O-methyl phosphorothioate (PS) antisense oligonucleotides (AON) (Fig. 1B).

\section{Skipping of Dysferlin Exon 32 is Efficient in Myoblasts}

Fibroblasts from a control subject were transduced by an inducible construct harbouring the MyoD gene and converted to fibroblastderived myoblasts upon induction of MyoD expression. They were transfected with the different AON (BP, ESE1, ESE2) combined with oligofectamine. RT-PCRs with forward primers in exon 30 and reverse primers in exon 33 evidenced a skipping of the DYSF-exon $32(\delta 32)$ with the AON targeting the predicted ESE sites (Fig. 2A). Two bands
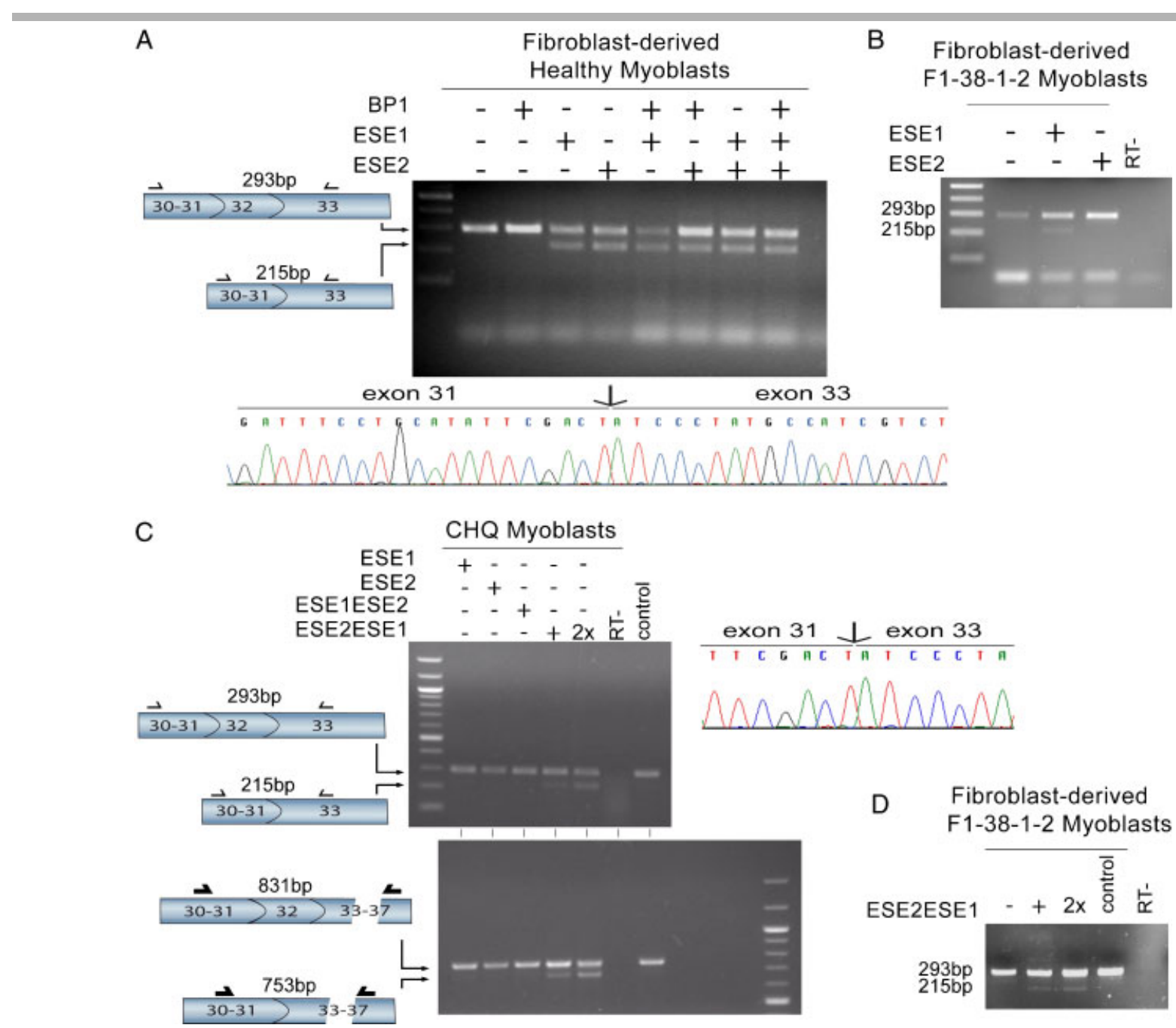

Figure 2. Dysferlin exon 32 skipping analysis. A: RT-PCR analysis of the transcript region flanking exon 32 (exons 30-33) in control fibroblastderived myoblasts. After treatment with AON ESE1 or ESE2, a shorter transcript fragment, not present in the control or RNA from cells treated with AON BP1, was observed at the expected size $(215 \mathrm{bp})$. Sequence analysis of the RT-PCR fragment identified the correct skipping of dysferlin exon 32 and the exact junction between exons 31 and 33. B: Fibroblast-derived myoblasts from Patient F1-38-1-2 were treated with both AON targeting ESE sequences. C: Infection of U7-smOPT-ESE2ESE1 at an MOI $\left(2.2510^{4}\right)$ in human control myoblasts (CHO) evidenced the same skipping as observed with AON although at a lower efficiency. When the MOI was doubled the signal was increased. Sequence analysis of the RT-PCR fragment shows the correct skipping of exon 32. No additional splice variants were observed when a PCR encompassing exon 30 to 37 (lower panel) was used instead of the 30-33 PCR. D: Transduction of patient F1-38-1-2 fibroblast-derived myoblasts with U7-smOPT-ESE2ESE1 at a MOI (2.25 104) evidenced the $\delta 32-D Y S F$ transcript. RT : RT-PCR reaction without RT: Control: positive reaction usina the full-lenath dvsferlin cDNA as a temblate. 
were detected, one corresponding to the wild-type transcript containing exon $32(293 \mathrm{bp})$ and another corresponding to the $\delta 32$ skipped mRNA (215bp), as confirmed by sequencing. No skipping was observed with the AON targeted against the lariat branchpoint only.

Subsequently, fibroblast-derived myoblasts from one patient (Patient F1-38-1-2: compound heterozygous c.3477C > A [exon 32, p.Tyr1159X] and c.5979dupA [exon 53, p.Glu1994ArgfsX3]) were treated as described above. Targeted transcriptional analysis of the patient's sample treated with ESE1, revealed a shorter transcript indicating the skipping of exon 32 (Fig. 2B). In contrast, no skipping was observed when ESE2 was used. An exon 32 containing transcript was identified from untreated fibroblastderived myoblasts from this patient, indicating it escaped the nonsense mediated mRNA decay mechanism although, in this situation, the intensity of the 293 bp product was lower. For ESE2, it is likely that, because the mutation is within its recognition site, this would confer a lower efficiency than the ESE1 AON. Therefore, due to this weak level of transcription, the skipped transcript was not observed.

\section{Lentiviral-Induced Skipping of Dysferlin Exon 32}

To confirm the possibility to skip exon 32 in patient's cells, we developed a lentiviral approach that would be more suited if a cellbased therapy using corrected myogenic stem cells appears as a valuable clinical approach, as already suggested for other diseases such as Duchenne muscular dystrophy [Benchaouir et al., 2007]. For this purpose, stem cell populations could be transduced with a lentiviral vector expressing the U7-snRNA containing antisense sequences designed to specifically skip exon 32 and restore dysferlin expression. Moreover, although the lentiviral based approach cannot be strictly extrapolated to other vectors and cell types, such a strategy could be used in different cell types expressing dysferlin, in particular monocytes, expressing a high level of dysferlin, which are easier and less invasive to obtain than myoblasts [Wein et al., 2009]. We designed four different lentiviral vectors harboring a cassette containing antisense sequences for the skipping of exon 32 from the dysferlin pre-mRNA. We used cassettes containing the selected sequences ESE1 and ESE2 either alone or in cis combination in the same vector (ESE1-ESE2 and ESE2-ESE1). To test whether this strategy would efficiently induce skipping of exon 32 in vitro, human control myoblasts (CHQ) were transduced with the U7 exon 32 lentiviral vectors. mRNAs were collected and analyzed by nested RT-PCR. A fragment corresponding to the expected size after skipping of exon 32 was observed from cells transduced with the U7smOPT-ESE2-ESE1 vector at a multiplicity of infection (MOI) of $2.2510^{4}$; this signal intensity was positively correlated with the MOI (Fig. 2C, upper panel). Efficient and specific skipping was further confirmed by direct sequencing of the RT-PCR products (Fig. 2C). No signal was observed in all other conditions (Fig. 2C). To ensure the specificity of exon 32 skipping, subsequent PCR experiments were conducted using primers in exons 30 (forward primer) and 37 (reverse primer) on the same samples. An identical pattern of skipping was observed without additional bands thus excluding aberrant splicing in the analyzed region (Fig. 2C lower panel). Identical results were obtained when the same lentivirus was used to transduce fibroblast-derived myoblasts from healthy control (data not shown).

Fibroblast-derived myoblasts obtained from patient F1-38-1-2 were then transduced by the U7smOPT-ESE2-ESE1 lentivirus vectors (Fig. 2D). Transduced cells expressed a significant amount of $\delta 32-D Y S F$ as revealed by RT-PCR.
Based on the "natural" proof of concept that an in frame deletion of exon 32 leads to a very late onset and mild phenotype according to Sinnreich and colleagues [2006], it is likely that exon 32 is at least partially dispensable, and can be deleted without major functional consequences. Moreover, in the patient reported by Sinnreich and colleagues [2006], the natural exon 32 skipped-allele partially complemented the null mutation in trans. In our opinion, this makes exon 32 the most pertinent target for exon skipping as a therapeutic approach in dysferlinopathies because no other in-frame deletions of single exons have ever been correlated to mild phenotypes. Here, not only do we show efficient exon 32 skipping in control pre-mRNA, but we also demonstrate that mutations in exon 32 can be removed from the patient's cells and that further synthesis of the $\delta 32$ skipped mRNA is not altered.

Other in-frame exon-skipping could ameliorate the phenotype severity, similar to the patient reported by Sinnreich and colleagues [2006]. This should be related to the corresponding DYSF encoded protein region, which would have been removed. Several reports have described patients with in-frame deletions, but most of them were associated with typical or severe phenotypes [De Luna et al., 2007; Therrien et al., 2006] making them a priori not pertinent as target exons (namely, exons 25 and 49) for therapeutic skipping. However, very few deletions of entire exon(s) have been identified in DYSF, and further explorations are needed to clarify their frequency. In a recent study of a cohort of patients affected with LGMD2B or Miyoshi myopathies in whom only one mutation was found, we have identified several internal deletions or duplications in DYSF [Krahn et al., 2009a]. This first study indicates that a systematic screening of deletions in DYSF, in particular in atypical mildly affected patients, would be of interest, and could lead to the identification of a larger number of in frame deletions. This would provide crucial information on potential exon skipping targets.

The modularity of dysferlin is still a matter of debate. However, a partial deletion of the $\mathrm{C} 2 \mathrm{D}$ domain in dysferlin suggests a certain level of functional redundancy. As for exon 32, which is included in the C2D domain, several in-frame exons encode part of other $\mathrm{C} 2$ domains and could be considered as potential targets for exon skipping. However, no natural proof of principle exists as yet and future studies will be needed to confirm these potentialities. In addition, other domains in dysferlin are essential and should never be targeted. This is the case of the unique C-terminus TM domain encoded by exon 54, whereas exons 53 and 54 could, in theory, be skipped with in frame maintenance.

Therefore, therapeutic exon-skipping in dysferlinopathies should be considered as a targeted, rather than a more general approach as is the case for dystrophinopathies [Beroud et al., 2007]. Indeed, mutational analyses in dysferlinopathies has revealed predominantly single nucleotide changes with no apparent mutational hot spots, and, except for rare cases, no phenotype to genotype correlations have been established so far. This situation is in contrast to dystrophinopathies where the milder consequences of deleting single or multiple in-frame exons in the dystrophin gene have been learned from Becker muscular dystrophy patients.

Currently, data from the Leiden Muscular Dystrophy pages ( database (www.dmd.nl) and our cohort of patients [Krahn et al., 2009a; unpublished data] include 13 patients carrying diseasecausing mutations in exon 32 , in a homozygous state in five patients, and in a compound heterozygous state in eight patients. Nine additional patients carry one disease-causing mutation in exon 32, whereas the second disease-causing mutation has not yet 
Table 1. Disease-Causing Nonsense or Frameshifting Mutations in Exon 32 of the Dysferlin Gene

\section{Number of reports}

\section{Mutation}

(Leiden Muscular Dystrophy pages` and our cohort)

\section{c.3444_3445delinsAA}

(p.Tyr1148X)

c. $3471 \mathrm{C}>\mathrm{A}$

(p.Tyr1157X)

c. $3477 \mathrm{C}>\mathrm{A}$

(p.Tyr1159X)

c. $3478 \mathrm{C}>\mathrm{T}$

(p.Gln1160X)

c.3516_3517del

(p.Ser1173X)

c.3517dupT

(p.Ser1173PhefsX2)

c.3518_3519insT

(p.Ser1173PhefsX2)

Total 8

Homozygous status 1

Compound heterozygous status 1

Identification in patients without identification

of a second disease-causing mutation 6

Total 2

Homozygous status 2

Compound heterozygous status 0

Identification in patients without identification of a second disease-causing mutation 0

Total 2

Compound heterozygous status 2

Identification in patients without identification of a second disease-causing mutation 0

Total 1

Homozygous status 1

Compound heterozygous status 0

Identification in patients without identification of a second disease-causing mutation 0

Total 5

Homozygous status 0

Compound heterozygous status 4

Identification in patients without identification of a second disease-causing mutation 1

Total 2

Homozygous status 0

Compound heterozygous status 1

Identification in patients without identification of a second disease-causing mutation 1

Total 2

Homozygous status 1
Homozygous status 0

Compound heterozygous status 0

Identification in patients without identification of a second disease-causing mutation 1

Mutational data are described using the nomenclature of the Human Genome Variation Society (www.hgvs.org/mutnomen). Nucleotide numbering reflects cDNA numbering with +1 corresponding to the A of the ATG translation initiation codon in the reference sequence (human DYSF, GenBank NM_003494.2), according to journal guidelines. The initiation codon is codon 1 .

been identified. Altogether, the disease-causing mutations identified in these patients include five different nonsense mutations and two different frameshifting mutations (Table 1). Diseasecausing nonsense or missense mutations as well as short insertions and/or deletions in DYSF exon 32 could be excised by using the exon 32-skipping approach with specific AON as reported here. Such a strategy could be applied to patients carrying these mutations either at a homozygous, or a compound heterozygous state in a future clinical trial.

\section{Acknowledgments}

We are very grateful to patients for their invaluable contribution to this study. We acknowledge Pierre Cau, Claire Navarro, Guillaume Precigout, Graziella Griffth, Arnaud Jollet, and Sebastien Courrier for their assistance in the experiments performed. Some of the cells used in this study were kindly provided by France Leturcq and Jamel Chelly from the Cochin Bio-Bank. We warmly thank Valérie Allamand for critical reading, discussions, and constructive comments on this manuscript, and the Myocastor study group for its constant support. This work was financially supported by the Association Française contre les Myopathies (AFM, France), the UPMC, CNRS, and the Institut National de la Santé et de la Recherche Médicale (INSERM, France), the Jain Foundation Inc., and the MYORES Network of Excellence (contract 511978) and MYOAMP
STREP (contract 037479), both from the European Commission 6th Framework Programme.

\section{References}

Aartsma-Rus A, Janson AA, Kaman WE, Bremmer-Bout M, van Ommen GJ, den Dunnen JT, van Deutekom JC. 2004. Antisense-induced multiexon skipping for Duchenne muscular dystrophy makes more sense. Am J Hum Genet 74:83-92.

Bansal D, Miyake K, Vogel SS, Groh S, Chen CC, Williamson R, McNeil PL, Campbell KP. 2003. Defective membrane repair in dysferlin-deficient muscular dystrophy. Nature 423:168-172.

Barde I, Zanta-Boussif MA, Paisant S, Leboeuf M, Rameau P, Delenda C, Danos O. 2006. Efficient control of gene expression in the hematopoietic system using a single Tet-on inducible lentiviral vector. Mol Ther 13:382-390.

Bashir R, Britton S, Strachan T, Keers S, Vafiadaki E, Lako M, Richard I, Marchand S, Bourg N, Argov Z, Sadeh M, Mahjneh I, Marconi G, Passos-Bueno MR, Moreira Ede S, Zatz M, Beckmann JS, Bushby K. 1998. A gene related to Caenorhabditis elegans spermatogenesis factor fer-1 is mutated in limb-girdle muscular dystrophy type 2B. Nat Genet 20:37-42.

Benchaouir R, Meregalli M, Farini A, D’Antona G, Belicchi M, Goyenvalle A, Battistelli M, Bresolin N, Bottinelli R, Garcia L, Torrente Y. 2007. Restoration of human dystrophin following transplantation of exon-skipping-engineered DMD patient stem cells into dystrophic mice. Cell Stem Cell 1:646-657.

Beroud C, Tuffery-Giraud S, Matsuo M, Hamroun D, Humbertclaude V, Monnier N, Moizard MP, Voelckel MA, Calemard LM, Boisseau P, Blayau M, Philippe C, Cossée M, Pagès M, Rivier F, Danos O, Garcia L, Claustres M. 2007. Multiexon skipping leading to an artificial DMD protein lacking amino acids from exons 45 through 55 could rescue up to $63 \%$ of patients with Duchenne muscular dystrophy. Hum Mutat 28:196-202.

Cartegni L, Krainer AR. 2003. Correction of disease-associated exon skipping by synthetic exon-specific activators. Nat Struct Biol 10:120-125.

Chaouch S, Mouly V, Goyenvalle A, Vulin A, Mamchaoui K, Negroni E, Di Santo J, Butler-Browne G, Torrente Y, Garcia L, Furling D. 2009. Immortalized skin fibroblasts expressing conditional $\mathrm{MyoD}$ as a renewable and reliable source of converted human muscle cells to assess therapeutic strategies for muscular dystrophies: validation of an exon-skipping approach to restore dystrophin in duchenne muscular dystrophy cells. Hum Gene Ther 20:784-790.

Charrier S, Stockholm D, Seye K, Opolon P, Taveau M, Gross DA, Bucher-Laurent S, Delenda C, Vainchenker W, Danos O, Galy A. 2005. A lentiviral vector encoding the human Wiskott-Aldrich syndrome protein corrects immune and cytoskeletal defects in WASP knockout mice. Gene Ther 12:597-606.

De Luna N, Freixas A, Gallano P, Caselles L, Rojas-Garcia R, Paradas C, Nogales G, Dominguez-Perles R, Gonzalez-Quereda L, Vilchez JJ, Márquez C, Bautista J, Guerrero A, Salazar JA, Pou A, Illa I, Gallardo E. 2007. Dysferlin expression in monocytes: a source of mRNA for mutation analysis. Neuromuscul Disord $17: 69-76$.

Desmet FO, Hamroun D, Lalande M, Collod-Beroud G, Claustres M, Beroud C. 2009. Human Splicing Finder: an online bioinformatics tool to predict splicing signals. Nucleic Acids Res 37:e67.

Edom F, Mouly V, Barbet JP, Fiszman MY, Butler-Browne GS. 1994. Clones of human satellite cells can express in vitro both fast and slow myosin heavy chains. Dev Biol 164:219-229.

Gorman L, Suter D, Emerick V, Schumperli D, Kole R. 1998. Stable alteration of premRNA splicing patterns by modified U7 small nuclear RNAs. Proc Natl Acad Sci USA 95:4929-4934.

Goyenvalle A, Vulin A, Fougerousse F, Leturcq F, Kaplan JC, Garcia L, Danos O. 2004. Rescue of dystrophic muscle through U7 snRNA-mediated exon skipping. Science 306:1796-1799.

Heemskerk HA, de Winter CL, de Kimpe SJ, van Kuik-Romeijn P, Heuvelmans N, Platenburg GJ, van Ommen GJ, van Deutekom JC, Aartsma-Rus A. 2009. In vivo comparison of $2^{\prime}$-O-methyl phosphorothioate and morpholino antisense oligonucleotides for Duchenne muscular dystrophy exon skipping. J Gene Med 11:257-266.

Krahn M, Beroud C, Labelle V, Nguyen K, Bernard R, Bassez G, Figarella-Branger D, Fernandez C, Bouvenot J, Richard I, Ollagnon-Roman E, Bevilacqua JA, Salvo E, Attarian S, Chapon F, Pellissier JF, Pouget J, Hammouda el H, Laforêt P, Urtizberea JA, Eymard B, Leturcq F, Lévy N. 2009a. Analysis of the DYSF mutational spectrum in a large cohort of patients. Hum Mutat 30:E345-E375.

Krahn M, Borges A, Navarro C, Schuit R, Stojkovic T, Torrente Y, Wein N, Pecheux C, Levy N. 2009b. Identification of different genomic deletions and one duplication in the dysferlin gene using multiplex ligation-dependent probe amplification and genomic quantitative PCR. Genet Test Mol Biomarkers 13:439-442.

Krahn M, Wein N, Lostal W, Bourg-Alibert N, Nguyen K, Courrier S, Vial C, Labelle V, De Petris D, Borges A, Mattei MG, Roudaut C, Miyake K, McNeil P, Cau P, Leturcq F, Bartoli M, Lévy N, Richard I. 2008. G.O.5 Partial functionality of a 
Mini-dysferlin molecule identified in a patient affected with moderately severe primary dysferlinopathy. Neuromusc Disord 18:781.

Liu J, Aoki M, Illa I, Wu C, Fardeau M, Angelini C, Serrano C, Urtizberea JA, Hentati F, Hamida MB, Bohlega S, Culper EJ, Amato AA, Bossie K, Oeltjen J, Bejaoui K, McKenna-Yasek D, Hosler BA, Schurr E, Arahata K, de Jong PJ, Brown Jr RH. 1998. Dysferlin, a novel skeletal muscle gene, is mutated in Miyoshi myopathy and limb girdle muscular dystrophy. Nat Genet 20:31-36.

Lu QL, Rabinowitz A, Chen YC, Yokota T, Yin H, Alter J, Jadoon A, Bou-Gharios G Partridge T. 2005. Systemic delivery of antisense oligoribonucleotide restores dystrophin expression in body-wide skeletal muscles. Proc Natl Acad Sci USA 102:198-203.

Naldini L. 1998. Lentiviruses as gene transfer agents for delivery to non-dividing cells. Curr Opin Biotechnol 9:457-463.

Nguyen K, Bassez G, Krahn M, Bernard R, Laforet P, Labelle V, Urtizberea JA, FigarellaBranger D, Romero N, Attarian S, Leturcq F, Pouget J, Lévy N, Eymard B. 2007. Phenotypic study in 40 patients with dysferlin gene mutations: high frequency of atypical phenotypes. Arch Neurol 64:1176-1182.

Sinnreich M, Therrien C, Karpati G. 2006. Lariat branch point mutation in the dysferlin gene with mild limb-girdle muscular dystrophy. Neurology 66:1114-1116.
Smith PJ, Zhang C, Wang J, Chew SL, Zhang MQ, Krainer AR. 2006. An increased specificity score matrix for the prediction of SF2/ASF-specific exonic splicing enhancers. Hum Mol Genet 15:2490-2508.

Therrien C, Dodig D, Karpati G, Sinnreich M. 2006. Mutation impact on dysferlin inferred from database analysis and computer-based structural predictions. J Neurol Sci 250:71-78.

Tuffery-Giraud S, Béroud C, Leturcq F, Yaou RB, Hamroun D, Michel-Calemard L, Moizard MP, Bernard R, Cossée M, Boisseau P, Blayau M, Creveaux I, Guiochon-Mantel A, de Martinville B, Philippe C, Monnier N, Bieth E, Khau Van Kien P, Desmet FO, Humbertclaude V, Kaplan JC, Chelly J, Claustres M. 2009. Genotype-phenotype analysis in 2,405 patients with a dystrophinopathy using the UMD-DMD database: a model of nationwide knowledge base. Hum Mutat 30:934-945.

Urtizberea JA, Bassez G, Leturcq F, Nguyen K, Krahn M, Levy N. 2008. Dysferlinopathies. Neurol India 56:289-297.

Wein N, Krahn M, Courrier S, Bartoli M, Salort-Campana E, Nguyen K, Fernandez C, Pouget J, Fossat C, Depetris D, Leturcq F, Cau P, Levy N. 2009. Immunolabelling and flow cytometry as new tools to explore dysterlinopathies. Neuromuscul Disord [Epub ahead of print]. 